Copyright by the American Physical Society. Lyman, E ; Schmittmann, B., Sep 2005. "Steady states of a nonequilibrium lattice gas," PHYSICAL REVIEW E 72(3) Part 2: 036127. DOI: 10.1103/PhysReve.72.036127.

PHYSICAL REVIEW E 72, 036127 (2005)

\title{
Steady states of a nonequilibrium lattice gas
}

\author{
Edward Lyman \\ Department of Environmental and Occupational Health and Department of Computational Biology and Bioinformatics, \\ University of Pittsburgh, Pittsburgh, Pennsylvania 15206, USA \\ B. Schmittmann \\ Center for Stochastic Processes in Science and Engineering and Department of Physics, \\ Virginia Tech, Blacksburg, Virginia 24061-0435, USA \\ (Received 19 April 2005; published 28 September 2005)
}

\begin{abstract}
We present a Monte Carlo study of a lattice gas driven out of equilibrium by a local hopping bias. Sites can be empty or occupied by one of two types of particles, which are distinguished by their response to the hopping bias. All particles interact via excluded volume and a nearest-neighbor attractive force. The main result is a phase diagram with three phases: a homogeneous phase and two distinct ordered phases. Continuous boundaries separate the homogeneous phase from the ordered phases, and a first-order line separates the two ordered phases. The three lines merge in a nonequilibrium bicritical point.
\end{abstract}

DOI: 10.1103/PhysRevE.72.036127

PACS number(s): 05.70.Ln, 05.10.-a, 05.65.+b

\section{INTRODUCTION}

The statistical treatment of systems driven far from equilibrium presents exciting theoretical challenges [1,2]. Lacking a unified understanding afforded equilibrium phenomena by the work of Boltzmann and Gibbs, we are exploring unknown territory without recourse to an established theory. Our physical intuition, developed in the context of equilibrium systems, can be misleading when faced with nonequilibrium problems. We therefore turn our attention to computational "experiments" in which a manifestly nonequilibrium state can be established and studied, seeking to identify key features shared by many nonequilibrium systems. While real physical systems are unquestionably important, complications and subtle details may obscure such common features. While motivated by realistic problems, microscopic rules and boundary conditions are chosen simple enough to facilitate a comprehensive computational study of the full parameter space. Many of the questions relevant in equilibrium remain interesting, especially as concern the nature of phase transitions and the principle of universality. The richness of nonequilibrium phenomena is often surprising, as the relaxation of the detailed balance constraint allows a variety of unexpected possibilities: in contrast to equilibrium, the $d y$ namics now affects the stationary (long-time) properties of the system. Particularly dramatic effects have been observed in models where the violation of detailed balance is combined with spatial anisotropies and dynamic conservation laws [1]. There, effective long-range interactions can be induced even if the microscopic rules are perfectly local in space and time [3].

In this paper, we consider a model from this class: namely, a lattice gas of two species of particles and holes on a fully periodic lattice in two spatial dimensions. To drive the system out of equilibrium, we bias the hopping rates of the two species in opposite directions, reminiscent of an "electric" field $E$ acting on opposite "charges" (though we stress that there is no Coulomb interaction). A nonzero charge current signals the nonequilibrium steady state. The two species interact through an excluded-volume constraint and nearestneighbor attractions. We choose the interactions carefully, in order to unify three important models which appear as limiting cases of our more general theory. First, by letting all particles attract each other, irrespective of their identity (charge), the nondriven limit corresponds to the familiar Ising lattice gas [4]. This well-known equilibrium model will serve as an anchor for our studies of driven systems. Turning the bias on but removing all members of one species, we recover the driven Ising lattice gas introduced by Katz, Lebowitz, and Spohn [5] (the KLS model). The third limit, obtained by letting the interaction strength vanish, corresponds to a noninteracting two-species model first proposed by Schmittmann, Hwang, and Zia [6] (the SHZ model). While the KLS model phase separates, via a continuous transition, into high- and low-density strips aligned with the field [5], the SHZ model orders into transverse, charge- and masssegregated, strips [6], similar to jamming instabilities in traffic models [7]. If a charge imbalance is imposed, these strips drift [8]. Our study will allow us to bridge the gap between these very different scenarios. To set the scene, we briefly survey some of the relevant previous work.

As a single-parameter modification of the Ising lattice gas, the KLS model is a minimal model for the study of nonequilibrium steady states (NESS's). Particle hops along one direction (parallel to the $x$ axis) occur at the normal equilibrium rate, as if in contact with a heat bath at temperature $T$. Particle hops in the other direction (parallel to the $y$ axis) are enhanced (suppressed) in the positive (negative) direction by coupling to an external field $E$. With periodic boundaries in the $y$ direction, a nonzero current is maintained, and the system settles into a NESS. At half-filling, there remains a continuous transition, though with $T_{c}(E)$ increasing monotonically with $E$ and saturating at $1.414 T_{c}(E$ $=0)$ [9-11]. The transition falls into a novel universality class with exponents distinct from the Ising ones [12]. The critical behavior is strongly anisotropic, with distinct sets of exponents characterizing fluctuations perpendicular and parallel 
to $E$. There has been some discussion regarding the nature of these fluctuations, with some authors disputing the original claim that the correct mesoscopic description is Gaussian in the perpendicular direction [13]. Though the anisotropy makes numerical investigations of the critical behavior quite subtle and computationally intensive, recent high-precision Monte Carlo studies compare the two mesoscopic descriptions. The results are in complete agreement with the predictions of the original field theory [9]. As a final note, we mention that the combination of anisotropic dynamics and a conservation law introduces power law correlations at all $T$ $>T_{c}$ [3], a manifestation of the relaxation of the detailed balance constraint. These correlations are revealed by the structure factor, which has a discontinuity singularity at the origin. In this sense, even the "disordered" phase is quite non-trivial.

Turning to multispecies versions, the simplest (SHZ) model [6] allows two different types of particles, distinguished only by their interaction with the external field. Positive (negative) particles are biased to hop in the positive (negative) $y$ direction and interact only via an excludedvolume constraint. The temperature is absorbed into $E$, and the only parameters are $E$, the overall mass density $m$, and the overall charge density (i.e., the density difference of the two species) $f$. Here, the mechanism for ordering is the mutual volume exclusion of the particles, so that at sufficiently strong $E$ and large $m$, the system locks into a high-density strip perpendicular to $E$, with positive and negative particles blocking each other. For nonzero charge density $f$, this strip is found to drift in the direction of the minority species [8]. Depending on where the phase boundary is crossed, firstorder or continuous transitions are observed [14,15]. Various other remarkable properties have been discovered. For a range of aspect ratios, configurations with nonzero winding number ("barber poles") are quite frequently observed, in addition to the usual transverse strips, raising the possibility of bistability [16]. Power law correlations characterize the disordered phase, with directionality-dependent exponents [17]. Another subtle issue concerns the lower critical dimension: While an exact solution for a strictly one-dimensional model, characterized by a single "lane" parallel to $E$, precludes a transition [18,19], Monte Carlo data for a "twolane" model indicate the presence of a macroscopic cluster in finite systems [20]. Very subtle finite-size effects control the decay of this cluster in the thermodynamic limit [21-23].

In this paper, we consider the two-species model at finite $T$ and $E$, where interparticle interactions are expected to play an important role. By varying $T, E$, and $f$, the fraction of the total population which are of the minority species, we can interpolate smoothly from the KLS model to the (noninteracting) two-species SHZ model. Hence, we expect a competition between the two types of ordered configurationsparallel versus transverse strips-favored by these two limits. As $f$ varies from 0.0 (KLS model) to 0.5 (equal numbers of each), there should be some critical $f$ where the preferred order switches. To explore these phenomena in more detail, we map out the phase diagram in $E, f$, and $T$ for a range of system sizes. The energy scale is set by our choice of the interparticle attraction $J$, and the overall mass density $m$ is fixed at 0.5 so that the Ising critical point remains ac- cessible. Many questions arise in connection with earlier work. How do nearest-neighbor attractions modify the twospecies transition? What will be the effect of a few "impurities" (i.e., minority particles) on the KLS transition? At what concentration do the impurities become relevant and change the nature of the transition? Preliminary results, focusing on a restricted parameter space, were already reported in [24]; here, we explore a much wider parameter range, including several system sizes. We will be able, if not to answer these questions fully, then to at least suggest the character of their resolution sufficiently to guide further research.

Our main results are as follows. At fixed $E$ and sufficiently small $f$, a line of continuous transitions emerges from the pure KLS $(f=0.0)$ point in the $f-T$ plane. This line separates the disordered phase from an ordered one, characterized by a particle-rich strip parallel to $E$. As we increase $f$, we encounter a bicritical point, where the transition line splits into a line of continuous order-disorder transitions, from disorder into a strip transverse to $E$, and a line of first-order transitions along which transverse and parallel order coexist. If we fix $f$ and lower $T$, we first observe the transition from disorder into the transverse strip, followed by a transition into parallel order. This topology persists at higher $E$, except that all lines are shifted to slightly higher temperatures. The size dependence of the phase diagram is subtle, since the main features are controlled by different scaling variables. On the one hand, the transition into the transverse strip is controlled by the effective drive $L_{y} E / T$ where $L_{y}$ is the system size in the drive direction. On the other hand, the bicritical point appears to depend on the scaling variable $L_{y} f$ which translates into the number of rows (transverse to $E$ ) which can be filled with the minority species. Finally, the pure KLS point requires finite-size scaling at fixed shape factor $A \propto L_{y} / L_{x}^{3}[10]$ in two spatial dimensions.

The remainder of the paper is organized as follows. We first describe in detail the microscopic model and the observables which are used to locate the different phases. We then present our simulation results, beginning with the structure of typical configurations in different parts of parameter space and their associated order parameters. By monitoring the signatures of first- and second-order transitions, we compile a cut through the phase diagram at fixed $E$, with variable $f$ and $T$. The phase boundaries and their dependence on system size are analyzed in some detail. To complete the picture, we present two cuts at different but fixed temperatures, crossing the phase boundaries by varying $E$ and $f$. We conclude with a brief summary and a discussion of some open questions.

\section{MICROSCOPIC MODEL AND OBSERVABLES}

We consider periodic square lattices of size $L_{x} \times L_{y}$, in two spatial dimensions, with $E$ parallel to the (positive) $y$ axis. A configuration is specified by the set of occupation variables, $\{\sigma(\mathbf{r})\}$, where $\sigma(\mathbf{r})$ takes three values, $\pm 1,0$ denoting a positive (negative) particle or a hole at lattice site $\mathbf{r}$. Often, we will only need to distinguish particles from holes via $n(\mathbf{r}) \equiv|\sigma(\mathbf{r})|$. All lattices are half filled-i.e., $m$ $\equiv\left(L_{x} L_{y}\right)^{-1} \sum_{\mathbf{r}} n(\mathbf{r})=1 / 2$ - so that the Ising critical point remains accessible. An important parameter is the fraction of 
negative particles (the "minority species") in the system: $f$ $=\left(m L_{x} L_{y}\right)^{-1} \sum_{\mathbf{r}} \delta_{-1, \sigma(\mathbf{r})}$. Clearly, we only need to consider the sector $0 \leqslant f \leqslant 0.5$, from having no negative particles at all to equal numbers of each species. For later reference, we also introduce the charge density $q \equiv\left(L_{x} L_{y}\right)^{-1} \Sigma_{\mathbf{r}} \sigma(\mathbf{r})=m-f$. The nearest-neighbor attraction is modeled by the Ising Hamiltonian

$$
H=-4 J \sum_{\left\langle\mathbf{r}, \mathbf{r}^{\prime}\right\rangle} n(\mathbf{r}) n\left(\mathbf{r}^{\prime}\right) .
$$

We choose attractive interactions $J>0$, regardless of species. While many other choices are possible and interesting, ours provides maximum linkage to known cases: Ising, KLS, and SHZ. The Monte Carlo dynamics conserves the number of each species and is specified as follows. An update attempt begins by picking a bond at random. If the bond connects a particle-hole pair, the contents are exchanged with the Metropolis rate $\min (1, \exp \{-[\Delta H-\delta y E \sigma(\mathbf{r})] / T\})$ [25]. Hence, at $E=0$ we recover the equilibrium Ising model with conserved magnetization, coupled to a heat bath at temperature $T$. We will set $J=1$ and measure $E$ in units of this (arbitrary) energy scale. $T$ will be quoted in units of the Onsager temperature, $T_{c}(E=0)$. The change in the $y$ coordinate, due to the proposed move, is denoted by $\delta y$, and $\Delta H$ is the associated change in internal energy. The term $\delta y E \sigma(\mathbf{r})$ models the gain or loss of energy from the coupling to $E$; if $\delta y \sigma(\mathbf{r})$ is positive (negative), the move is favored (unfavored). Our model, in which $E$ and $T$ are varied independently, raises an interesting issue. If the ratio $E / T$ is quite large, it becomes almost impossible for particles to hop backwards. In a finite system, this implies that a relatively small fraction of the minority species-provided the "right" fluctuation occurs-is sufficient to form a stable blockage. Even though such a fluctuation becomes less probable in a larger system, the dynamics nevertheless becomes nonergodic in the limit $E / T \rightarrow \infty$. In principle, this can be avoided by introducing, e.g., a small probability for particles to exchange places [15]. To limit the number of parameters, we circumvent these problems here by considering different initial configurations and a range of system sizes.

The dynamics is diffusive and therefore conserves both charge and mass density. Though the local effect of the external field is analogous to the effect of an electrostatic potential on electric charges, the boundary conditions exclude the possibility of a global Hamiltonian description.

As overall density is conserved, we expect ordered configurations to be strips of higher density coexisting with strips of lower density. We therefore introduce the Fourier transform of the local mass variable,

$$
\widetilde{s}\left(m_{x}, m_{y}\right) \equiv \frac{\pi}{L_{x} L_{y}} \sum_{x, y} n(x, y) e^{2 \pi i\left(m_{x} x / L_{x}+m_{y} y / L_{y}\right)},
$$

which is labeled by (integer) wave numbers $m_{x}=0$, $1, \ldots, L_{x}, m_{y}=0,1, \ldots, L_{y}-1$. The structure factor

$$
S\left(m_{x}, m_{y}\right) \equiv\left\langle\left|\widetilde{s}\left(m_{x}, m_{y}\right)\right|^{2}\right\rangle
$$

then serves as a good order parameter, since it is sensitive to mass-segregated strip configurations. For example, $S(1,0)$ will be $O(1)$ for a strip aligned with the field, characteristic of KLS order; similarly, $S(0,1)$ will detect a strip transverse to $E$ which develops in the SHZ (two-species) model; and both are normalized to $O\left(1 / L_{x} L_{y}\right)$ for a disordered configuration. We also monitor a "susceptibility,"-i.e., the fluctuations of the order parameter:

$$
\Delta\left(m_{x}, m_{y}\right) \equiv L_{x} L_{y}\left[\left\langle\left|\widetilde{s}\left(m_{x}, m_{y}\right)\right|^{4}\right\rangle-\left\langle\left|\widetilde{s}\left(m_{x}, m_{y}\right)\right|^{2}\right\rangle^{2}\right] .
$$

We note that $S\left(m_{x}, m_{y}\right)$ involves the Fourier transform of the mass variable and is therefore not sensitive to any chargesegregated structures. Replacing $n(x, y)$ by $\sigma(x, y)$ in Eq. (3) generates structure factors which respond to charge inhomogeneities. We have monitored these and their fluctuations throughout, and found that their behavior is consistent with the mass-based quantities.

When $S$ is calculated, the average is taken over multiple steady-state configurations of a Monte Carlo run, with a typical run lasting $8 \times 10^{5}$ Monte Carlo steps (MCS) and $2 L_{x} L_{y}$ bond update attempts per MCS. Data are collected every 400 MCS; fluctuations of observables indicate that this interval is sufficient to produce uncorrelated data in the largest (60 $\times 80$ ) systems considered. Typically, the initial $2 \times 10^{5}$ of the total $8 \times 10^{5}$ MCS are discarded to ensure that data are taken from the steady state. Near critical points and at low temperatures these numbers require modification, due to long correlation times and long-lived metastable states. In such cases the only recourse is a careful analysis of individual, very long runs. When that is necessary we will measure a quantity closely related to $S$ :

$$
s\left(m_{x}, m_{y}\right) \equiv\left|\widetilde{s}\left(m_{x}, m_{y}\right)\right|^{2},
$$

which measures the type of order present in a single configuration. We can then track $s$ for different $m_{x}$ 's and $m_{y}$ 's over the course of a run and see precisely how the averages are generated.

Now that we have described the various quantities which will be used to probe the behavior of our model, we turn to the presentation of the data.

\section{RESULTS}

\section{A. Phase diagram in $f$ and $T$}

In this section we seek the location and character of transitions by scanning in $f$ and $T$ at fixed drive $E$. We choose $E=2.0$ since this intermediate value still allows for a significant fraction of backward jumps, thus avoiding the spurious metastable configurations discussed above. At the same time, it is large enough to induce measurable currents and other clear signatures of far-from-equilibrium behavior. The two order parameters $S(1,0)$ and $S(0,1)$ and their fluctuations are monitored in order to identify the different phases. Large peaks in their fluctuations, or the presence of hysteresis, are used as indicators of continuous versus first-order transitions, respectively. For clarity, we first present a quick overview of the topology of the phase diagram and then turn to the details of the data which underlie this picture.

Figure 1 shows the phase diagram in the $f$ - $T$ plane, at $E$ $=2.0$, for two different system sizes. Three phases are found: 


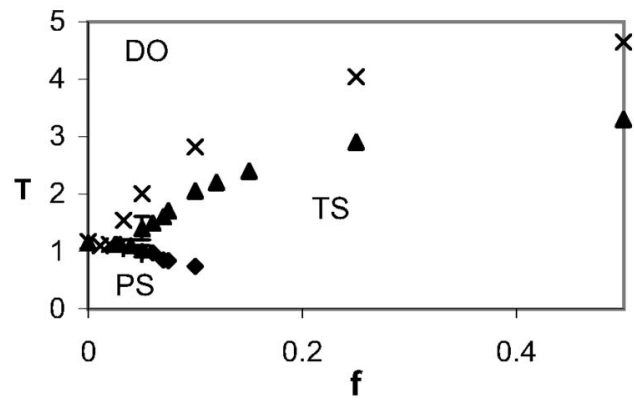

FIG. 1. Phase diagram in $f$ and $T$ for $E=2.0$. Triangles and diamonds are boundaries in the $40 \times 40$ system; $\times$ 's are for the $60 \times 60$ system.

a homogeneous, disordered phase (DO), a transverse strip (TS) phase as in the two-species model, and a parallel strip (PS) phase as in the KLS model. The value of $f$ determines which phase is observed: at $f=0$ there is only one species of particles, and a single transition is observed from disorder into the parallel strip. As $f$ increases, this transition persists until the number of the minority species is sufficient to create a blockage and form the transverse strip. From here on, two transitions are observed: from disorder into the transverse strip and at a lower temperature from the transverse strip into the parallel strip. The shift of the DO-TS strip will be addressed in a later section. Upon increasing $f$ further, only the DO-TS transition can be detected. Since the PS and TS phases are not connected by a continuous symmetry transformation, the TS-PS line cannot have a critical end point. However, for $f>0.10$ it occurs at such a low temperature that it cannot be observed in simulations of a reasonable length.

After this brief discussion of the phase diagram, we consider the phases and their boundaries in more detail. We begin with some pictures of typical configurations at various points in the phase diagram. All frames show $40 \times 40$ lattices, with $E$ pointing up. White (black) pixels are positively (negatively) biased particles; blue-green (gray in print) pixels are holes.
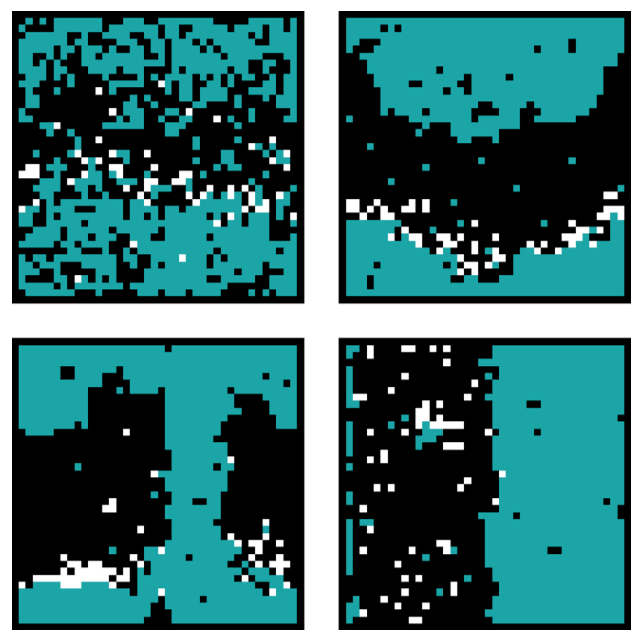

FIG. 2. (Color online) Configurations of the $40 \times 40$ lattice at $f=0.075, E=2.0$ for four temperatures. Upper left, $T=1.77$; upper right, $T=0.95$; lower left, $T=0.84$; lower right, $T=0.78$.

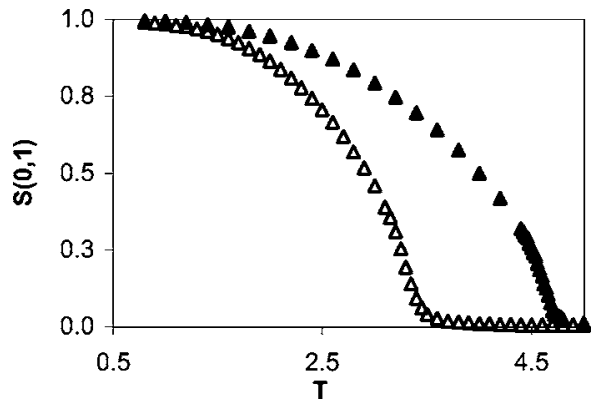

FIG. 3. $S(0,1)$ as a function of $T$ for $f=0.50, E=2.0$. Open (solid) triangles are for the $40 \times 40(60 \times 60)$ system.

To illustrate the presence of three phases, we select $f$ $=0.075$ (Fig. 2), where only 1.5 rows of the minority species are present. At high temperatures, the system is disordered; no figure is shown. The first frame shows a configuration just below criticality at $T=1.77$. Though a blockage can form, the strip is not longer symmetric with respect to + and - . This leads to a drifting of the strip: Occasionally, the rather thin blockage of the minority species is opened by backward hops, and the majority species pours through. These particles then travel quite rapidly around the periodic lattice and attach to the back of the majority blockage, the net result being an upward drift of the strip. Lowering $T$ further to 0.84 (third frame) it appears that interfaces parallel to $E$ are becoming favorable; this type of configuration is common at these intermediate values of $f$ : here, parallel and transverse strips compete with each other. Indeed the final frame $(T=0.78)$ shows the preferred low-temperature configuration: a single strip of mixed charge parallel to $E$, suggesting a sequence of two transitions as a function of $T$.

Next, we map out order parameters and their fluctuations as a function of $T$, for a range of $f$ and two system sizes, in order to locate the phase boundaries. While this cannot replace a high-precision finite-size scaling analysis of the transitions, it is intended to provide a first glance of how the fluctuations scale with system size.

We begin at $f=0.50$, with equal numbers of each type of particle. In Figs. 3 and $4, S(0,1)$ and its fluctuations are plotted as a function of $T$ for two system sizes $(40 \times 40$ and $60 \times 60) ; S(1,0)$ is not shown as the only observed transition here is from the homogeneous phase into the transverse strip. In both systems, $S(0,1)$ goes smoothly to zero as $T$ is increased. A clean peak in $\Delta(0,1)$ is also observed in each

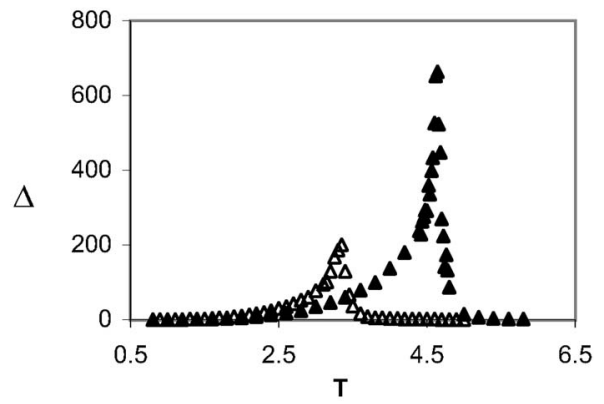

FIG. 4. $\Delta(0,1)$ as a function of $T$ for $f=0.50, E=2.0$. Open (solid) triangles are for the $40 \times 40(60 \times 60)$ system. 


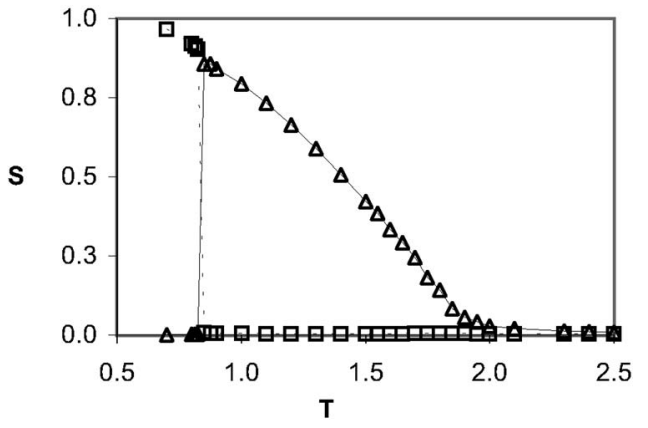

FIG. 5. $S(0,1)$ and $S(1,0)$ as a function of $T$ for $f=0.075, E$ $=2.0$ in the $40 \times 40$ system. Triangles [squares] are for $S(0,1)$ $\times[S(1,0)]$. Lines and dashes are provided to guide the eye.

system, increasing in amplitude with the system size. These two observations are consistent with a continuous transition into the transverse strip at $f=0.50$, and we therefore use the location of the peak in $\Delta(0,1)$ to locate the phase boundary in Fig. 1, with $T_{c}(L=40)=3.35$ and $T_{c}(L=60)=4.64$.

Upon reducing $f$ we observe a sequence of two transitions as a function of $T$. Figure 5 shows both order parameters $S(0,1)$ and $S(1,0)$ for 1.5 rows of the minority species: $f$ $=0.075$ in the $40 \times 40$ system. Also shown in Fig. 6 is $\Delta(0,1)$ for 1.5 rows of the minority species in both the $40 \times 40$ and $60 \times 60$ systems. We have omitted the $60 \times 60$ data for the order parameters to keep the plot uncluttered. As before, $S(0,1)$ and $\Delta(0,1)$ signal a continuous transition into the TS phase as $T$ is lowered, though the signal in $\Delta(0,1)$ is much more pronounced in the $60 \times 60$ system. And also as before, there are significant fluctuations associated with this phase, due to strip drifting. Specifically, in the $60 \times 60$ system $\Delta(0,1)$ actually has a broad secondary peak in the ordered phase. The large magnitude of this signal is quite unexpected and awaits a satisfactory explanation. For now, we only note that lowering $T$ increases the effective bias, $E / T$, and therefore enhances fluctuations associated with the drive.

Lowering $T$ further we observe $S(0,1)$ falling abruptly, while $S(1,0)$ climbs rapidly, suggesting a discontinuous transition from the transverse strip into the parallel strip. In the neighborhood of such a transition, one expects to see metastability of the unfavored phase, and this is indeed the case as shown in Fig. 7. Here we have plotted time traces (as opposed to configurational averages) of structure factors for individual configurations, $s(1,0)$ and $s(0,1)$, defined in Eq.

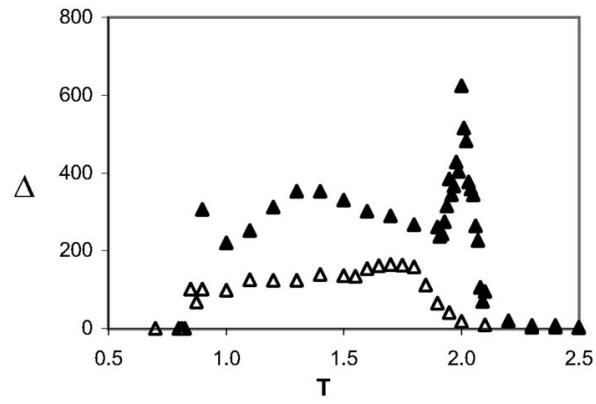

FIG. 6. $\Delta(0,1)$ as a function of $T$ for $f=0.075, E=2.0$. Open (solid) triangles are for the $40 \times 40(60 \times 60)$ system.

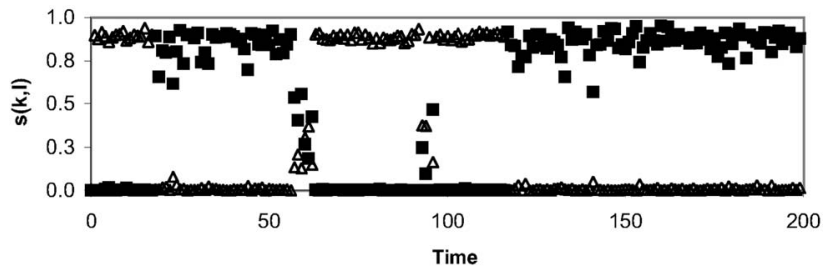

FIG. 7. Time trace at $T=0.832, E=2.0, f=0.075$, in the 40 $\times 40$ system. Time in units of $2 \times 10^{5} \mathrm{MCS}$ is plotted on the horizontal axis. The values of $s(0,1)$ and $s(1,0)$ (triangles and squares, respectively) are plotted on the vertical axis.

(5). When $s(1,0)[s(0,1)]=1$ the configuration is a perfect PS (TS). Sufficiently close to the transition, the time traces reveal the expected behavior, as the system switches between the two ordered phases. Notice the length of the run shown: $4 \times 10^{7} \mathrm{MCS}$, which is a factor of 40 longer than typical runs, indicating that the lifetimes of metastable configurations are already quite long even in the $40 \times 40$ system, rendering such behavior nearly unobservable in the $60 \times 60$ system.

At smaller values of $f$ we are nearing the junction of the three phase boundaries, which considerably complicates the analysis of data from small systems in a couple of ways. The sequence of transitions (DO-TS followed by TS-PS) becomes difficult to resolve, as they are quite close in temperature, and massive fluctuations from the first-order TS-PS line may wash out the signal in $\Delta(0,1)$ which locates the continuous DO-TS line. And if the junction of the three lines is indeed a nonequilibrium bicritical point, we can expect finite-size effects to interfere with the analysis. We can, however, make some progress based on the assumption that the relevant control parameter near the bicritical point is the number of rows of the minority species. This hypothesis will be treated in more detail below in the sections on scaling arguments.

At precisely one row of the minority species it is no longer possible to accurately resolve the two transitions in the $40 \times 40$ system. A weak transverse ordering is observed, with $S(0,1)$ reaching at most $40 \%$ of perfect order. In the vicinity of the PS-TS first-order transition, huge fluctuations associated with switching between metastable configurations are observed, which wash out the signal of the DO-PS transition. However, it is interesting that these transitions can be resolved in larger systems at precisely one row of the minority species: There, these two transitions are sufficiently far apart in temperature since (as we will see below) $T_{c}$ increases with $L_{y}$ across the DO-TS transition. This likely explains why the DO-TS transition is not observed in the $40 \times 40$ system.

Below one row of the minority species we no longer observe the transverse order, though we caution that this may be strictly correct only for the finite system. With $f$ just below a single row of the minority species, the hightemperature phase is homogeneous and the low-temperature phase is the parallel strip. In the vicinity of the transition, huge fluctuations are observed in $S(0,1)$ and especially $S(1,0)$, where the fluctuations are an order of magnitude larger than the signal at the DO-TS boundary. In this region 
neither $S(1,0)$ nor $S(0,1)$ posesses a well-defined average; time traces indicate vigorous competition between the two ordered phases. We conjecture that we are close to the bicritical point in the finite system and are therefore unable to resolve the transition without some knowledge of scaling to guide the analysis. At smaller $f$ we are farther from the bicritical point, and the complications from the presence of the minority species are less severe. $S(1,0)$ (data not shown) indicates that the low-temperature configuration is a single parallel strip, with a smooth approach to zero, again suggesting a continuous transition. Time traces and data for $\Delta(1,0)$ are consistent with this conjecture.

We close this section on the $f$ - $T$ phase diagram with a summary of the results. The picture at higher $f$ is clear: a clean continuous transition into the horizontal strip, with $T_{c}$ decreasing with $f$. When $f$ is reduced to approximately three rows of the minority species, the signal of the transition remains clear, though it now sits atop a shoulder of fluctuations of the ordered phase. At yet smaller $f$, a second transition appears between the two ordered phases at lower $T$; it has the characteristics of a first-order transition. At even lower $f$, at approximately one row of the minority species, both fluctuations perpendicular and parallel to $E$ become so violent that the DO-TS transition is only seen in larger systems, as the two transitions nearly overlap in the $40 \times 40$ system. Close to the $f=0$ point, the transition is once again clean and apparently continuous into a vertical strip of mixed charge. Of course, all these statements are based on an analysis of finite systems. In order to draw robust conclusions, a more systematic analysis of larger samples is required.

\section{B. Phase diagram in $f$ and $E$}

In the preceding section we studied a slice of the phase diagram at constant $E$, varying the fraction of the minority species and the temperature. Varying $T$ effectively varies both the strength of particle-particle attractions and the strength of the bias, since the relevant quantities in the rates are $J / T$ and $E / T$. In this section we consider a different cut through the phase diagram. By varying $E$ and $f$ at fixed $T$, the interparticle attractions are held constant while the strength of the bias is varied. In this way we can study directly the competition between the drive and attractive interactions. In the following, we choose a value for $T$ and then scan in $E$ for several values of $f$. The temperatures are chosen by reference to the KLS temperature: $T=2.0$ is above the critical temperature of the KLS model at saturation, and $T$ $=1.2$ is at the critical temperature for $E=2.0$, studied in the previous section. At this stage, we have only data for 40 $\times 40$ systems and are therefore as yet unable to speculate on results for larger systems. However, they cast a new light on the more detailed results of the previous sections.

$f-E: T=2.0$. As before, we first survey the phase diagram with the help of some typical configurations. The qualitative picture will then be made more quantitative in the next section by examining the behavior of order parameters and their fluctuations.

Figure 8 shows a series of configurations at various $f$ for $E=20.0$. The first frame clearly shows the transverse strip at
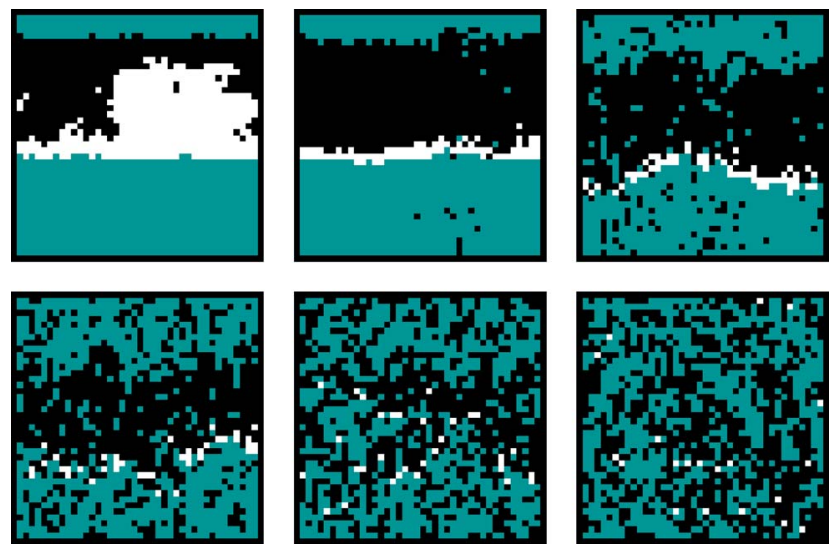

FIG. 8. (Color online) Configurations for several $f$ at $E=20.0$, $T=2.0$, in the $40 \times 40$ system. Upper left, $f=0.50$; upper middle, $f$ $=0.10$; upper right, $f=0.075$; lower left, $f=0.05$; lower middle, $f$ $=0.04$; lower right, $f=0.025$.

$f=0.50$, and the absence of travelers suggests that the strip is stationary. In the next frame we have reduced $f$ to 0.10 , reducing the thickness of the minority species to exactly two rows. Consequently we now see some travelers trickling through a break in the blockage. Watching an animation in this region of the phase diagram reveals an interesting behavior: the strip is mostly quiescent, except for a few particles hopping back and forth at the particle-hole interface. Aside from the different ratio of + to - , these configurations look similar to the $f=0.50$ strip. Then, a sudden large fluctuation opens up a hole in the minority blockage: the + particles pour through, and the strip fluctuates and drifts partway around the lattice, until the blockage is reestablished. Reducing $f$ further to 0.075 (third frame) we see a strip in the middle of one of these fluctuation events. In contrast to $f=0.10$ where such large fluctuations are relatively rare, the situation is now reversed; i.e., the quiescent periods become less frequent. In the fourth frame, we set $f=0.05$, and while the strip is still clearly visible it now drifts continuously. The final two frames show $f=0.04$ and 0.025 . Now there is no longer any clear evidence of phase separation. This rough picture is consistent with our earlier investigation of the two-species transition, where we observed transverse order at and above a single row of the minority species.

The pictures in Fig. 8 are summarized in Fig. 9, which presents the phase diagram at $T=2.0$. The boundary separates a PS at high $E$ and $f$ from DO at small $E$ and $f$. As we are above the critical temperature for the KLS model at saturation bias, the vertical strip does not appear at any $E$; at low $f$ (where we might otherwise expect to see such ordered configurations) the system simply remains disordered for any $E$ and $f$. Figures 10 and 11 show $S(0,1)$ and $\Delta(0,1)$ for several values of $f$; in the interest of clarity $\Delta(0,1)$ is plotted for only four representative $f$ 's. As $E$ is increased the system orders into a transverse strip, with $S(0,1)$ saturating at smaller values as $f$ is decreased. At $f=0.05$ (exactly one row of the minority species) $S$ saturates at only 0.28 , indicating that the transverse ordering is rather weak, though comparison with the data for $f=0.025$ shows dramatically different 


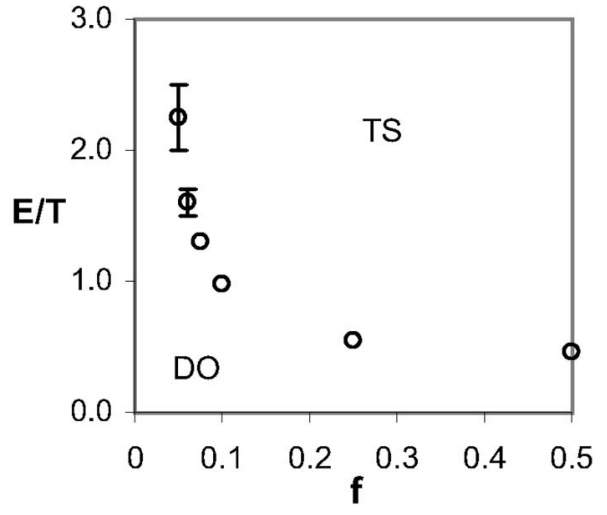

FIG. 9. Phase diagram in $f$ and $E / T$ at $T=2.0$, in the $40 \times 40$ system. Disorder (DO) is observed at small $f$ and $E$; the transverse strip (TS) dominates at high $f$ and $E$. The error bars are smaller than the size of the data points, unless indicated.

behavior. Here $S(0,1)$ reaches a maximum of only 0.01 , and the behavior can hardly be called "saturation." $\Delta(0,1)$ also signals a transition, though the clean, sharp spike at $f=0.50$ becomes a broad bump at $f=0.05$, and shows no signal at $f=0.025$. The susceptibility also indicates a difference in the ordered phases at different $f$ : at large $f$ increasing $E$ suppresses fluctuations, while at smaller $f$ (when the strip begins drifting) increasing $E$ enhances fluctuations. It is important to note that the fluctuations at high $E$ are fluctuations about the ordered phase, as $\Delta(0,1)$ is always $2-3$ orders of magnitude larger than $\Delta(1,0)$. Though not included in the plots, we have checked the behavior of the ordered phase for $E$ as high as 40. The fluctuations for small $f(f<0.10)$ saturate and bounce around a well-defined average, while for larger $f$ they are suppressed. This is true for $f=0.05$ and greater; at $f$ $=0.025$ the magnitudes of the fluctuations in either direction are comparable.

$f-E: T=1.2$. To observe the emergence of the KLS phase, we lower the temperature to $T=1.2<T_{K L S}$ and explore the corresponding $(f-E)$ slice of the phase diagram (Fig. 12). As long as we remain at $f>0.10$, we observe a transition similar to the one at $T=2.0$ : from the homogeneous phase into twospecies order. In contrast, for $f<0.04$ the KLS transition is observed, since the minority species is too scarce to form a blockage and $T<T_{K L S}(E=\infty)$. Between these two limiting values of $f$ we are again in the vicinity of the bicritical point,

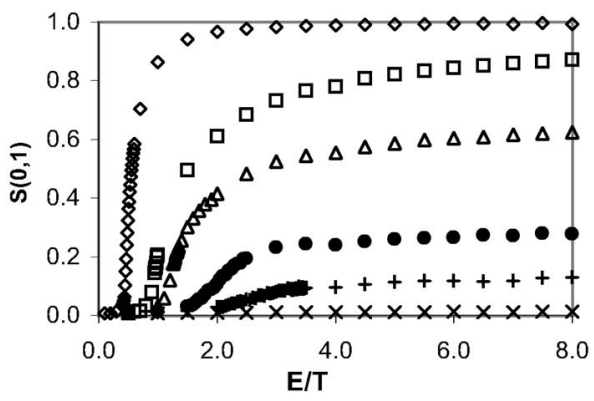

FIG. 10. $S(0,1)$ as a function of $E / T$ for several $f$ in the 40 $\times 40$ system: $f=0.50$, diamonds; $f=0.10$, squares; $f=0.075$, triangles; $f=0.05$, circles; $f=0.04$, + 's; $f=0.025$, $\times$ 's.

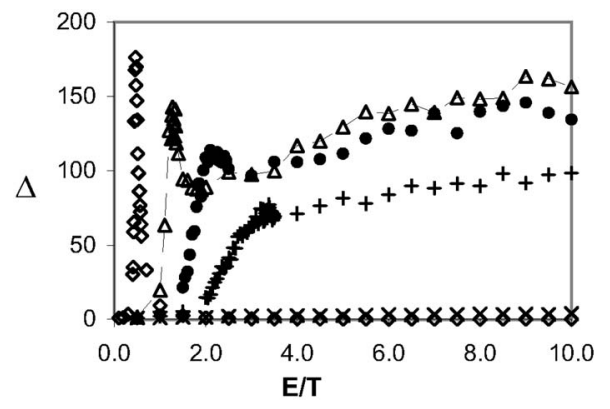

FIG. 11. $\Delta(0,1)$ as a function of $E / T$, in the $40 \times 40$ system, for several $f$. The symbols are the same as in the previous figure. A line has been added to the $f=0.075$ data to guide the eye.

and the situation becomes complicated due to the competing types of order.

Figure 13 shows typical configurations at two different values of $f$ for various $E$. For $f>0.075$ these configurations look much as they did at $T=2.0$, so they need not be included. The $f=0.075, E=20.0$ configuration shows some very interesting structure, almost "equal parts" KLS and twospecies order, suggesting competition between the two phases. We stress that this is a typical configuration. When $f$ is reduced to a single row $(f=0.05)$ this competition is reduced, and we see instead a KLS phase with some local two-species order. This trend continues upon reducing $f$ to zero, where at high $E$ the KLS order is observed. The other panels show $f=0.075$ and 0.05 at smaller values of $E, E$ $=4.0$ and 2.4, respectively. These $E$ values were chosen because they maximize the two-species order for these $f$ 's. In each case the strip drifts rapidly around the lattice. Interestingly, as the majority species is piled onto the back of the drifting strip it builds long fingers, leading to a very irregular interface.

In Fig. 14 we plot $S$ for the high- $f$ phases. For $f=0.50$ and $0.10, S(0,1)$ shows the system ordering into the two-species phase much as in the previous section. At $f=0.075$ the behavior changes dramatically. After maximizing the twospecies order at $E=3.0$, increasing $E$ further suppresses $S(0,1)$ and enhances $S(1,0)$, until both saturate below 0.2 . These values are hardly consistent with any kind of distinct

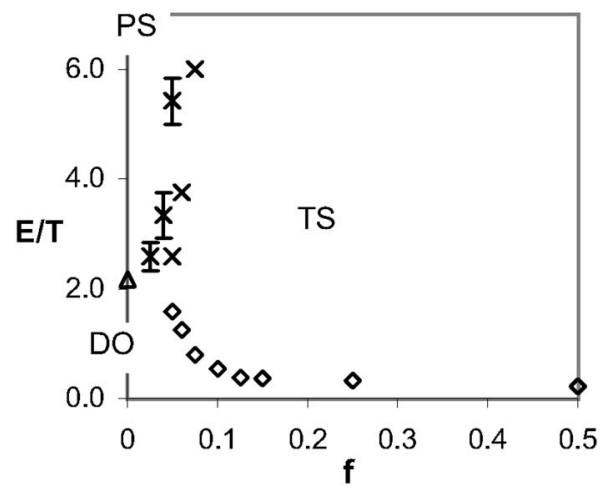

FIG. 12. Phase diagram in $E / T$ and $f$ for $T=1.2$, in the 40 $\times 40$ system. A continuous boundary separates DO from TS (diamonds) as well as DO from PS (triangles). $X$ 's indicate a possible boundary between TS and PS. A few typical error bars are shown. 

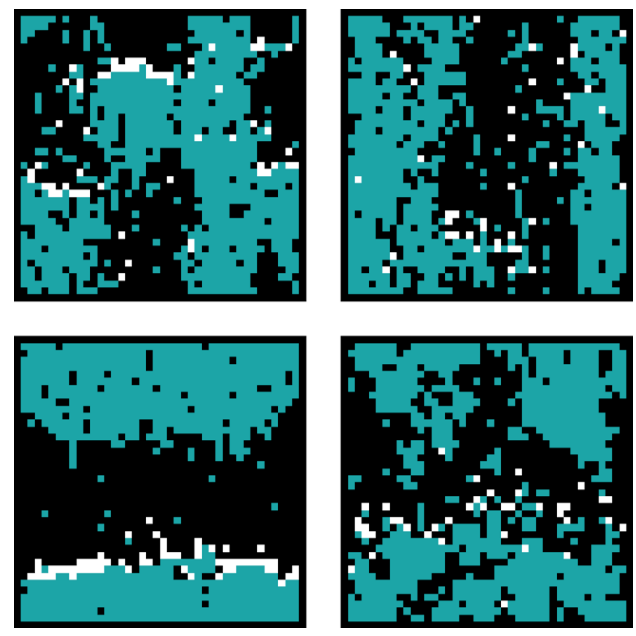

FIG. 13. (Color online) Typical configurations for the $T=1.2$ plane, in the $40 \times 40$ system. Upper left, $f=0.075, E=20.0$; upper right, $f=0.05, E=20.0$; lower left, $f=0.075, E=4.0$; lower right, $f$ $=0.05, E=2.4$.

order; nor do they indicate disorder. Instead, the system finds itself in configurations similar to the top left panel of Fig. 13: The competition between the two ordered phases seems very balanced in this small system. It would be interesting to simulate larger systems and explore whether this type of "phase competition" persists or whether the KLS order eventually becomes stable.

Additional information is provided by the susceptibilities. Observations of $\Delta(0,1)$ are consistent with a continuous transition from disorder into the transverse strip at small $E$. Seeking a signature of the KLS phase, we show $\Delta(1,0)$ and $\Delta(0,1)$ for $f=0.075$ in Fig. 15. Here we observe a second peak in $\Delta(0,1)$ at $E=7.2$, which corresponds to $S(0,1)$ $\simeq 0.4$ in Fig. 14. This peak is rather broad, and its amplitude is more than twice that of the first peak (associated with the DO-TS transition). Perhaps it suggests a first-order transition which would be observed in a larger system, separating the two-species phase from the KLS phase. Finally, at higher $E$ both $\Delta(0,1)$ and $\Delta(1,0)$ fluctuate around nonzero values, reflecting the fluctuations of the competing phases. We note no signal of a transition in $\Delta(1,0)$.

For $f$ in the neighborhood of 0.05 , the transverse strip is observed at low $E$ and the parallel strip at high $E$. The

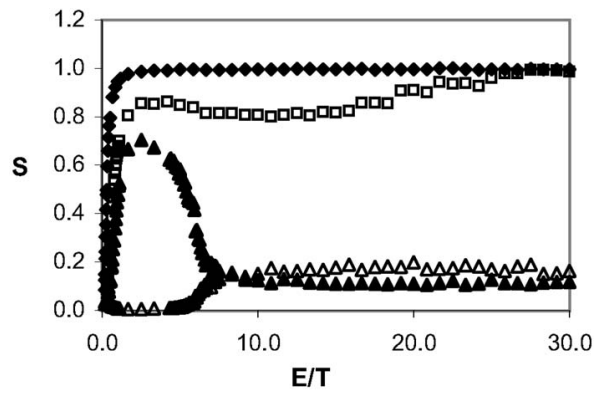

FIG. 14. $S(0,1)$ as a function of $E / T$, in the $40 \times 40$ system for several $f . f=0.50$, diamonds; $f=0.10$, squares; $f=0.075$, solid triangles. Open triangles indicate $S(1,0)$ for $f=0.075$.

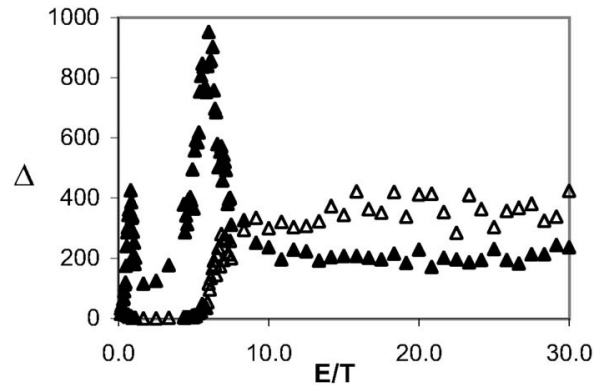

FIG. 15. $\Delta(0,1)$ (solid triangles) and $\Delta(1,0)$ (open triangles) as a function of $E / T$ in the $40 \times 40$ system for $f=0.075$.

boundary, however, is difficult to locate precisely due to the phase competition. Upon reducing $f$ below 0.05 we find that the transverse strip has essentially disappeared. Meanwhile, $S(1,0)$ saturates at 0.50 , indicating that the KLS strip has formed. Apparently the system is unable to completely order at any $E$, since $T_{K L S}(E=\infty)=1.4$. We stress that this behavior is due to the proximity of the KLS phase transition, not due to some residual competition with the transverse strip.

\section{Scaling arguments}

Now that we have surveyed the phase diagram in some detail, we turn to look closely at some of the boundaries with the help of some scaling arguments. Considering Fig. 1 again, we note two potentially troubling features. First, there is a shift in $T_{c}$ across the DO-TS boundary of about $50 \%$ between the two system sizes. We will characterize this shift using a mean-field scaling argument. Second, we note that the bicritical point has shifted towards the $f=0$ axis in the larger system. It has been alluded to before that the number of rows of the minority species, rather than the fraction $f$, might be the controlling variable. We will investigate this suggestion more carefully by considering some larger systems and rectangular geometries. Finally we study the $f=0$ phase transition, using scaling arguments developed for the KLS model.

The shift in $T_{c}$ with system size is most pronounced at $f$ $=0.50$. Previous work on the two-species model with $J=0$ treated the ordered phase in a mean-field approximation by solving equations of motion for the two different charge densities $[8,14]$. It was found that the scaling functions depend on the combination $E L_{y} / T$, indicating that the effective bias $E / T$ introduces a new length scale. This scaling implies an infinite-volume limit in which $E / T \rightarrow 0$ as $L_{y} \rightarrow \infty$, while keeping $E L_{y} / T$ fixed. Earlier analyses of the ordered phase based on these ideas have worked quite well, so that we now attempt to extend this approach to analyze quantities near criticality and for $J \neq 0$. There is no reason to expect success a priori, as both critical fluctuations and nonzero $J$ may modify the scaling variables and the mean-field exponents. In Figs. 16 and 17 we have plotted $S(0,1)$ and $\Delta(0,1)$ for $J=1.0$. [We have divided $\Delta(0,1)$, Eq. (4), by the volume in order to compare different system sizes more easily.] Rather than crossing the phase boundary by varying $T$ we have opted instead to vary $E$ since this allows us to vary the ef- 


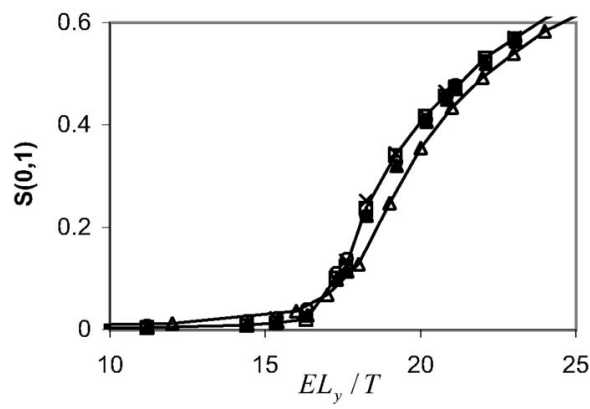

FIG. 16. $S(0,1)$ as a function of effective drive for $J=1, f$ $=0.50$. System sizes: $40 \times 40$, open triangles; $40 \times 60$, circles; 40 $\times 80$, $\times$ 's; $60 \times 60$, solid triangles; $60 \times 80$, squares. Lines have been added to the largest and smallest systems to guide the eye.

fective bias $E / T$ at constant interaction strength, $J / T$. While the collapse of $S(0,1)$ in Fig. 16 is not perfect, the meanfield scaling argument accounts for most of the shift in $T_{c}$. There is a shift in the peak of $\Delta(0,1)$ of about $3.6 \%$ between the largest and smallest systems; if the same data were plotted without rescaling, the shift in $T_{c}$ is about $52 \%$. Also of note is the extremely weak dependence on the transverse dimension, as predicted by the scaling argument.

Another issue concerns the location of the junction of the three phase boundaries, shown in Fig. 1. It is clear that the junction moves toward the $f=0$ axis as the system size is increased. In fact, in both systems the boundaries merge just below a single row of the minority species, which naturally corresponds to a smaller $f$ in the larger system. In Fig. 18 we have replotted the data from Fig. 1, replacing $f$ with $f L_{y} / 2$, which is simply the number of rows of the minority species. Near the junction of the three lines we have also included results from a few other system sizes with rectangular geometries. Plotted vs $f L_{y} / 2$, the junctions of the boundaries coincide, within the error bars, for all system sizes, suggesting that the onset of the two-species order occurs, at least in relatively small finite systems, when there are sufficient minority particles to form a single row. The crucial question concerns the extrapolation of this result to an appropriate thermodynamic limit. If the system size goes to infinity in the most naive way-i.e., $L_{x}, L_{y} \rightarrow \infty$ at fixed aspect ratio $L_{x} / L_{y}$-the particle density associated with a "single row" vanishes. It is possible that the DO-PS transition exists in an infinite volume only at $f=0$ and any finite density of "disor-

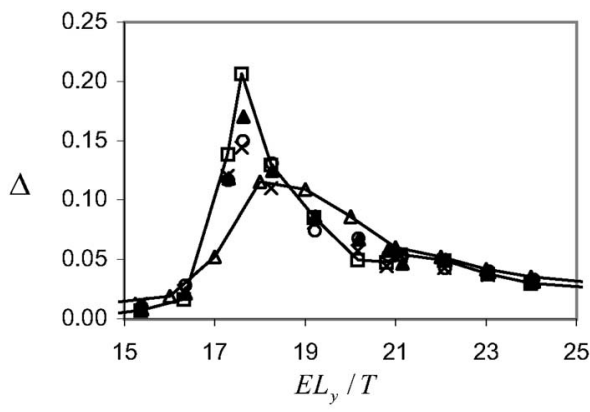

FIG. 17. $\Delta(0,1)$ as a function of effective drive for $J=1, f$ $=0.50$. The symbols are the same as in the previous figure.

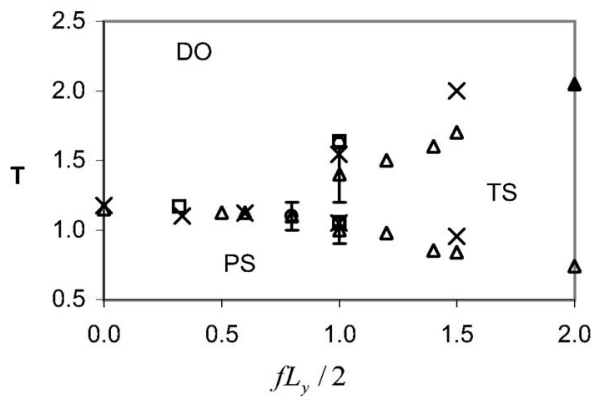

FIG. 18. The phase diagram in the $f$ - $T$ plane, $E=2.0$, with $f$ rescaled to represent the number of rows of the minority species. System sizes: solid triangles, $40 \times 40$; $\times$ 's, $60 \times 60$; squares, 60 $\times 80$.

der" (i.e., the minority species) induces the two-species order. Preliminary studies [26] indicate that the minority species does indeed constitute a relevant perturbation to the KLS fixed point. We will have to leave discussion of this issue to future work and for now limit ourselves to statements about finite systems.

At $f=0$ there is only one species and we observe the KLS transition at finite $E$. Though a great deal of study has been devoted to this transition at infinite $E$, there has been no detailed work at finite $E$. Here we present a basic finite-size scaling (FSS) analysis of this transition in order to locate $T_{c}(E=2.0)$ and also to demonstrate the subtleties which can arise when studying phase transitions with anisotropic, nonequilibrium dynamics.

Field-theoretic studies of the KLS model [12] indicate that the critical behavior is strongly anisotropic, meaning that correlation lengths diverge with different exponents in the field direction and perpendicular to the field. Specifically, the fluctuations perpendicular to the field are Gaussian $\left(\nu_{\perp}\right.$ $=1 / 2)$ while those parallel to the field are not $\left(\nu_{\|}=3 / 2\right)$. Correlations therefore grow faster in the parallel direction as $T \rightarrow T_{c}(E)$, suggesting an analysis of rectangular samples such that the anisotropic aspect ratio $A \equiv L_{\|}^{\nu_{\perp} / \nu_{\|}} L_{\perp}^{-1}$ is held fixed [10]. While there is some discussion regarding the correct mesoscopic model [13], detailed numerical simulations show that the exponents cited above are the correct ones $[9,11]$. In the following we will use only the phenomenological result of Leung for the scaling of the order parameter at fixed $A$ :

$$
S\left(T, L_{\|}, L_{\perp}\right)=L_{\|}^{-\beta / \nu_{\|}} \bar{S}\left(t L_{\|}^{1 / \nu_{\|}, L_{\|}^{\nu_{\perp}} / \nu_{\|}} L_{\perp}^{-1}\right),
$$

where $S$ refers to $S(1,0)$. A detailed discussion of the subtleties of the FSS analysis for the KLS model and precision numerical results can be found in [9]. Figure 19 presents our data for the scaled order parameter at $E=2$; the same data for saturation $E$ can be found elsewhere [27]. These data are not intended as a test of the mesoscopic model, merely meant to indicate that the exponents at infinite $E$ are consistent with those at finite $E$ and to determine $T_{c}(E=2.0)=1.20(2)$. The data collapse is comparable to that seen at saturation $E$, with the high-temperature (lower) branch collapsing quite well and the low-temperature (upper) branch showing small, but 


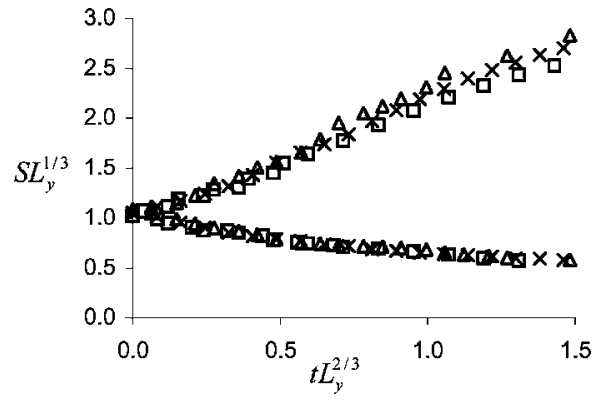

FIG. 19. Anisotropic scaling plot of $S(1,0)$ at $f=0, E=2.0$. System sizes: squares, $24 \times 54$; $\times$ 's, $28 \times 86$; triangles, $32 \times 128$.

systematic deviations from scaling. These deviations remain unexplained. They are possibly due to corrections to scaling or perhaps the asymptotic region is only observed very close to $T_{c}$.

We have seen how subtle are the issues surrounding the transition at $f=0$. Perhaps a fruitful way to proceed when $f$ $\neq 0$ is to adopt the technique introduced by Caracciolo et al. [28], directly measuring finite-volume correlation lengths for various geometries and volumes. In this way we may develop some understanding of how to approach the infinitevolume limit in a simple way, minimizing corrections to scaling which would complicate an uninformed analysis.

\section{CONCLUSIONS AND OUTLOOK}

We have compiled a detailed phase diagram for a system of two species of particles, interacting via attractive Ising interactions and driven into opposite directions by an external "electric" field $E$. The purpose of our analysis was to unify previous studies which were restricted either to just one species or to having only excluded-volume interactions. In the former case, particles order into a single strip aligned with the field direction, while in the second model, the two oppositely driven species form a jam in the shape of a transverse strip. We monitor structure factors, their fluctuations, and, if necessary, their time traces to identify the location and character of the transitions. Most of our data are taken at fixed $E$, varying the fraction $f$ of the "minority" species and temperature $T$. At high $f$, we observe a continuous transition from a disordered phase into the transverse strip, as the temperature is lowered. Noting a significant system-size dependence of the critical line, we invoke a mean-field scaling argument $[8,14]$ which suggests that $E L_{y} / T$ is a good scaling variable. This is confirmed quite satisfactorily by our data. At smaller $f(0.05 \leqslant f \leqslant 0.10$ for a $40 \times 40$ system $)$ we observe two transitions as $T$ is lowered: first into the transverse strip (continuous) and then into the parallel strip (first order). And finally at the smallest $f$ a single, continuous transition is observed in the parallel strip. The junction of the three phases-disorder, transverse, and parallel strip-appears to be a multicritical point. Analyzing the data for a range of system sizes suggests that its location scales with $f / L_{x}$, rather than $f$; i.e., the relevant quantity is the number of rows which can be formed by the minority particles. Depending on how the thermodynamic limit is approached, the multicritical point can shift to $f=0$.

Several projects suggest themselves to extend this work. First, an analysis of larger systems at fixed $T$ could clear up some questions, especially regarding the fate of the "phase competition" which is observed close to the multicritical point. Many of the "transitions" in the $40 \times 40$ system require an analysis of larger lattices in order to confirm their existence. Second, a look at structure factors in the disordered phase is likely to reveal the presence of long-range correlations. Since these are known to be quite distinct in the KLS [3] and SHZ [17] models, it would be interesting to investigate how the crossover occurs. Such a study would also yield considerable insight into the type of noise terms which would have to be added to the mean-field equations in order to capture fluctuations and critical properties accurately. These equations would then provide a reliable starting point for an analysis of the KLS transition in the presence of a few minority charges, in order to understand the true nature of the KLS critical point: does it mark the beginning of a critical line, or is it a multicritical point?

\section{ACKNOWLEDGMENTS}

We thank R.K.P. Zia, I. Georgiev, U.C. Täuber, A. Gambassi, M. Gubinelli, G. Korniss, and H.K. Janssen for fruitful discussions. This work was supported in part by NSF Grant Nos. DMR-0088451, DMR-0414122, and SBE-0244916, as well as the Jeffress Memorial Trust, Grant No. J-594.
[1] B. Schmittmann and R. K. P. Zia, in Phase Transitions and Critical Phenomena, edited by C. Domb and J. L. Lebowitz (Academic, London, 1995), Vol. 17.

[2] J. Marro and R. Dickman, Non-Equilibrium Phase Transitions in Lattice Models (Cambridge University Press, Cambridge, England, 1999).

[3] M. Q. Zhang, J-S. Wang, J. L. Lebowitz, and J. L. Vallès, J. Stat. Phys. 52, 1461 (1988); P. L. Garrido, J. L. Lebowitz, C. Maes, and H. Spohn, Phys. Rev. A 42, 1954 (1990); G. Grinstein, J. Appl. Phys. 69, 5441 (1991); B. Schmittmann and R. K. P. Zia, J. Stat. Phys. 91, 525 (1998).
[4] E. Ising, Z. Phys. 31, 253 (1925); B. M. McCoy and T. T. Wu, The Two-Dimensional Ising Model (Harvard University Press, Cambridge, MA, 1973).

[5] S. Katz, J. L. Lebowitz, and H. Spohn, Phys. Rev. B 28, 1655 (1983); J. Stat. Phys. 34, 497 (1984).

[6] B. Schmittmann, K. Hwang, and R. K. P. Zia, Europhys. Lett. 19, 19 (1992).

[7] O. Biham, A. A. Middleton, and D. Levine, Phys. Rev. A 46, R6124 (1992); D. Chowdhury, L. Santen, and A. Schadschneider, Phys. Rep. 329, 199 (2000).

[8] K-t. Leung and R. K. P. Zia, Phys. Rev. E 56, 308 (1997). 
[9] S. Caracciolo, A. Gambassi, M. Gubinelli, and A. Pelissetto, J. Phys. A 36, L315 (2003); J. Stat. Phys. 115, 281 (2004).

[10] K-t. Leung, Phys. Rev. Lett. 66, 453 (1991); Int. J. Mod. Phys. C 3, 367 (1992).

[11] J-S. Wang, J. Stat. Phys. 82, 1409 (1996); K-t. Leung and J-S. Wang, Int. J. Mod. Phys. C 10, 853 (1999).

[12] H. K. Janssen and B. Schmittmann, Z. Phys. B: Condens. Matter 64, 503 (1986); K-t. Leung and J. L. Cardy, J. Stat. Phys. 44, 567 (1986); 45, 1087(E) (1986).

[13] B. Schmittmann, H. K. Janssen, U. C. Täuber, R. K. P. Zia, K.-t. Leung, and J. L. Cardy, Phys. Rev. E 61, 5977 (2000).

[14] I. Vilfan, R. K. P. Zia, and B. Schmittmann, Phys. Rev. Lett. 73, 2071 (1994).

[15] G. Korniss, B. Schmittmann, and R. K. P. Zia, Europhys. Lett. 32, 49 (1995); J. Stat. Phys. 86, 721 (1996).

[16] K. E. Bassler, B. Schmittmann, and R. K. P. Zia, Europhys. Lett. 24, 115 (1993).

[17] G. Korniss, B. Schmittmann, and R. K. P. Zia, Physica A 239, 111 (1997).

[18] D. Mukamel, in Soft and Fragile Matter, edited by M. E. Cates and M. R. Evans (IOP, Bristol, 2000).

[19] B. Derrida, S. A. Janowsky, J. L. Lebowitz, and E. R. Speer,
Europhys. Lett. 22, 651 (1993); J. Stat. Phys. 73, 813 (1993). [20] J. T. Mettetal, B. Schmittmann, and R. K. P. Zia, Europhys. Lett. 58, 653 (2002).

[21] Y. Kafri, E. Levine, D. Mukamel, G. M. Schütz, and J. Török, Phys. Rev. Lett. 89, 035702 (2002).

[22] B. Schmittmann, J. T. Mettetal, and R. K. P. Zia, in Computer Simulation Studies in Condensed Matter Physics XVI, edited by D. P. Landau, S. P. Lewis, and H.-B. Schüttler (Springer, Heidelberg, 2003).

[23] I. T. Georgiev, B. Schmittmann, and R. K. P. Zia, Phys. Rev. Lett. 94, 115701 (2005).

[24] E. Lyman and B. Schmittmann, J. Phys. A 35, L213-L218 (2002).

[25] N. Metropolis, A. W. Rosenbluth, M. M. Rosenbluth, A. H. Teller, and E. Teller, J. Chem. Phys. 21, 1087 (1953).

[26] B. Schmittmann (unpublished).

[27] Edward Lyman and B. Schmittmann, in Recent Developments in Simulation Studies in Condensed Matter Physics XVI, edited by D. P. Landau, S. P. Lewis, and H.-B. Schüttler (Springer, Heidelberg, 2004).

[28] S. Caracciolo, A. Gambassi, M. Gubinelli, and A. Pelissetto, Eur. Phys. J. B 20, 255 (2001). 